\title{
Deux protéines supplémentaires au pays décidément hétérogène des LGMD !
}

Les dystrophies musculaires constituent un groupe d'affections génétiques extrêmement hétérogène caractérisées par une faiblesse et une atrophie progressive des muscles squelettiques. La topographie de l'atteinte initiale dépend de l'affection mais nombre d'entre elles débutent par une atteinte des muscles proximaux (ceintures pelvienne et scapulaire). Ainsi, par abus de langage et par facilité, ces dystrophies musculaires ont été rebaptisées LGMD (Limb Girdle Muscular Dystrophy ou myopathies des ceintures) de type 1 , pour les formes à transmission dominante ou de type 2 pour celles dont la transmission est récessive (Tableau I) $\left(\mathrm{m} / \mathrm{s} \mathrm{1996,} n^{\circ} 12, p .1445\right)$ [1]. Malgré l'explosion de gènes déjà impliqués, certaines de ces affections restaient cependant orphelines. Deux nouveaux gènes, déterminant les formes LGMD1C et LGMD2B, viennent "d'adopter " certaines de ces maladies orphelines [2-5].

C'est en parallèle que deux myopathies de topographie très différente ont été localisées dans le même inter- valle $(2 p 12-14)$ : une myopathie des ceintures, appelée LGMD2B, non associée à une modification de tout le complexe des sarcoglycanes, à la différence des formes de type $2 \mathrm{C}$ à 2F, et la myopathie de Miyoshi, caractérisée par une atteinte beaucoup plus distale débutant vers la fin de la deuxième décennie et touchant notamment le muscle gastrocnémien. La course à la protéine a abouti à une même cible, appelée dysferline pour sa ressemblance avec une protéine déjà identifiée chez Caenorhabditis elegans. Les mutants du gène correspondant, le gène fer-1, présentent dans cette espèce, une spermatogenèse anormale avec mobilité réduite des spermatozoïdes. La dysferline comprend une région de ciblage membranaire et, dans sa partie cytoplasmique, 4 domaines homologues des motifs C2. Les motifs C2, retrouvés dans de nombreuses protéines, ont la capacité de lier le calcium et les phospholipides à l'instar de la protéine kinase C. Le transcrit correspondant $(7 \mathrm{~kb})$ est exprimé dans le muscle squelettique, le cœur,

Tableau I

CLASSIFICATION DES LGMD

\begin{tabular}{|lcl|}
\hline Nomenclature & Localisation chromosomique & Protéine responsable \\
\hline LGMD1A & $5 \mathrm{q}$ & \\
LGMD1B & $1 \mathrm{q} 11-21$ & \\
LGMD1C & $3 \mathrm{p} 25$ & Cavéoline-3 \\
LGMD2A & $15 \mathrm{q} 15.1-\mathrm{q} 21.1$ & Calpaïne \\
LGMD2B & $2 \mathrm{p} 12-2 \mathrm{p} 14$ & Dysferline \\
LGMD2C & $13 q 12$ & $\gamma$-sarcoglycane \\
LGMD2D & $17 \mathrm{q} 12$ & $\alpha$-sarcoglycane \\
LGMD2E & $4 \mathrm{q} 12$ & $\beta$-sarcoglycane \\
LGMD2F & $5 \mathrm{q} 33$ & $\delta$-sarcoglycane \\
\hline
\end{tabular}

$m / s n^{\circ} 2$, vol. 15 , féurier 99 le placenta et, moins fortement, dans le foie, le poumon, le rein et le pancréas. Une forme plus courte $(4 \mathrm{~kb})$ est également présente dans le cerveau. Bien que ce transcrit soit également détecté par RT-PCR dans le testicule humain, aucune baisse de la fertilité n'a été rapportée chez les patients porteurs de LGMD2B. Les mutations observées chez les patients sont essentiellement non sens (délétions, duplications, mutations ponctuelles créant un codon stop) pour les formes homozygotes, et des mutations faux sens pour les formes hétérozygotes. Elles sont retrouvées dans les deux types d'affection déjà mentionnés ainsi que dans une troisième entité clinique, une myopathie distale avec atteinte privilégiée du tibial antérieur. Au vu des résultats présentés, il semble qu'une seule mutation puisse conduire à deux phénotypes différents, parfois même au sein d'une même fratrie. La fonction de la protéine humaine et le lien l'unissant potentiellement à la protéine du ver restent à découvrir. Parmi les protéines à l'origine des LGMD déjà découvertes, seule la calpaïne-3 $(\mathrm{m} / \mathrm{s}$ $1995, n^{\circ} 4, p .637$ ) semblait jouer un rôle à part, sans lien direct avec tout le complexe des autres protéines associées à la dystrophine. La dysferline pourrait ajouter une nouvelle catégorie fonctionnelle à ce groupe d'affections si hétérogène qu'il faudra peut-être en revisiter la nosologie...

Les cavéolines sont des protéines généralement associées aux cavéoles, structures formant de petites invaginations membranaires à la surface des cellules et qui seraient notamment capables de concentrer les événements de signalisation ayant lieu à 
la surface de la membrane plasmique. La cavéoline-3 est une forme spécifiquement musculaire de cavéoline. Elle est capable d'homo-dimérisation et d'hétérodimérisation avec une grande variété d'autres molécules. La cavéoline 3 est copurifiée avec le complexe dystrophine [6] et interagit directement avec la NO synthase neuronale, cette liaison entraînant la perte de l'activité NOS [7]. Le gène humain est localisé en 3 p25. L'analyse du gène de la cavéoline 3 chez des patients porteurs de LGMD à transmission dominante a permis de révéler un petit nombre de mutations, déterminant la forme LGMD1C. Les mutations détectées sont des mutations faux-sens ou une délétion ne perturbant pas le cadre de lecture, et la plupart sont localisées dans la région impliquée dans l'homo-oligomérisation de la protéine. Peut-on réellement lier cette affection à celles perturbant le com- plexe dystrophine? Il est encore trop tôt pour l'affirmer. En effet, d'après des expériences menées par l'équipe de K. Campbell qui, rappelons-le, est à l'origine de la découverte du groupe de protéines liées à la dystrophine $\left(m / s\right.$ 1998, $\left.n^{\circ} 4, p .519\right)$, la cavéoline 3 ne ferait pas partie intégrante du complexe comme les sarcoglycanes ou les dystroglycanes [8]. De plus, chez les patients porteurs de mutations du gène de la cavéoline 3 , aucune perturbation de l'expression des autres protéines n'est notée, ce qui est très différent des LGMD de type $2 \mathrm{C}$ à $2 \mathrm{~F}$ ou de la dystrophie musculaire de Duchenne, dans lesquelles toutes les sarcoglycanes ( $\alpha$ à $\delta$ ) sont effondrées. C'est donc toute la physiopathologie de ces affections qui reste à découvrir.
1. Duclos F, Broux O, Lim L, et al. $\beta$-Sarcoglycane : une protéine du complexe dystrophine-glycoprotéines est responsable d'une forme récessive de dystrophie des ceintures. Med Sci 1995; 11: 1732-8.

2. Liu J, Aoki M, Illa et al. Dysferlin, a novel skeletal muscle gene, is mutated in Miyoshi myopathy and limb girdle muscular dystrophy. Nat Genet 1998 ; 20 : 31-6.

3. Bashir R, Britton S, Strachan T et al. A gene related to Caenorhabditis elegans spermatogenesis factor fer-1 is mutated in limb-girdle muscular dystrophy type 2B. Nat Genet 1998; 20: 37-42.

4. Minetti C, Sotgia F, Bruno C et al. Mutations in the caveolin-3 gene cause autosomal dominant limb-girdle muscular dystrophy Nat Genet 1996; $18: 365-8$.

5. Mc Nally EM, de SA Moreira E, Duggan DJ, et al. Caveolin-3 in muscular dystrophy. Hum Mol Genet $1998 ; 7: 871-7$

6. Song KS, Scherer PE, Tang ZL, et al. Expression of caveolin-3 in skeletal, cardiac, and smooth muscle cells. J Biol Chem 1996 ; 271 : 15160-5.

7. Venema VJ, Ju H, Zou R, Venema RC. Interaction of neuronal nitric-oxide synthase with caveolin-3 in skeletal muscle. J Biol Chem 1997; 45 : 28187-90.

8. Crosbie RH, Yamada H, Venzke DP, Lisanti MP, Campbell KP. Caveolin-3 is not an integral component of the dystrophin glycoprotein com-

H.G. plex. FEBS Lett 1998 ; 427: 279-82 